\title{
Immunotherapy Benefit in a Patient With Non- Small Cell Lung Cancer and a Rare BRAF Mutation
}

\author{
Rebekah Rittberg ${ }^{1}$, Shantanu Banerji ${ }^{2}$, Susan Green ${ }^{3}$, Gefei Qing ${ }^{4}$, David E. Dawe ${ }^{2}$ \\ 1. Oncology and Hematology, University of Manitoba, Winnipeg, CAN 2. Oncology and Hematology, CancerCare \\ Manitoba, University of Manitoba, Winnipeg, CAN 3. Oncology and Hematology, CancerCare Manitoba, Winnipeg, \\ CAN 4. Pathology, University of Manitoba, Winnipeg, CAN
}

Corresponding author: David E. Dawe, ddawe@cancercare.mb.ca

\begin{abstract}
Immunotherapy is less effective in non-small cell lung cancer (NSCLC) with driver mutations in epidermal growth factor receptor (EGFR) or anaplastic lymphoma kinase $(A L K)$ and some may extrapolate this trend to other driver mutations. Up to $4 \%$ of NSCLC cases contain a BRAF mutation. Most BRAF mutations are V600E, and little is known about the impact of treatment in rare BRAF G469A mutations. We present a case of a patient found to have BRAF G469A mutated NSCLC. She was diagnosed with Stage IIIB NSCLC and treated with concurrent chemotherapy and radiation. Post-treatment imaging demonstrated disease progression and she was started on nivolumab, resulting in a dramatic and prolonged response which is ongoing after 76 cycles. Her substantial response and prolonged benefit suggest that BRAF-mutated NSCLC may respond better than $E G F R$ - or $A L K$-driven disease to immunotherapy. Due to the rarity of specific mutations, this case adds to the limited current published literature on NSCLC harbouring a BRAF G469A mutation and suggests that immunotherapy is a reasonable treatment option.
\end{abstract}

Categories: Pathology, Oncology, Pulmonology

Keywords: immune checkpoint inhibitor, driver mutation, non-small cell lung cancer, braf, nivolumab

\section{Introduction}

The therapeutic approach to non-small cell lung cancer (NSCLC) has dramatically shifted with identification of targetable driver mutations and the introduction of immune-checkpoint inhibitors (ICI) [1]. Treatment algorithms for tumors possessing epidermal growth factor receptor (EGFR) mutations and anaplastic lymphoma kinase $(A L K)$ translocations involve targeted tyrosine kinase inhibitors then cytotoxic chemotherapy, before considering ICI monotherapy due to concerns about poor efficacy of ICI in the driver mutation-activated population [2]. Other activating mutations have been identified, including the BRAF gene, representing potential sites for direct acting or downstream therapeutic targets where the impact of ICI is not known [3].

Received 06/08/2020

\section{Case Presentation}

Here we present a case of a 61-year-old female, previous smoker, diagnosed with Stage IIIB NSCLC, adenocarcinoma subtype, programmed death-ligand 1 (PD-L1) $>50 \%, A L K$ translocation, $E G F R$ mutation, and KRAS mutation-negative and de novo BRAF G469A mutated. She was originally treated on a clinical trial with radical radiation (66 Gray in 33 fractions) and concurrent chemotherapy (cisplatin and etoposide), randomized to receive metformin in combination. Post-treatment computed tomography (CT) demonstrated improvement of her right hilar mass, but new 6.0 x $4.4 \mathrm{~cm}$ left adrenal mass and $3.1 \times 2.5 \mathrm{~cm}$ right adrenal mass (Figure 1). She started nivolumab alone, with near-complete response after 33 cycles. She has received 76 cycles of nivolumab with ongoing disease control more than four years later. Her treatment has been complicated with Grade 1 diverticulitis and recurrent Grade 1 pneumonitis which has required steroid treatment. 

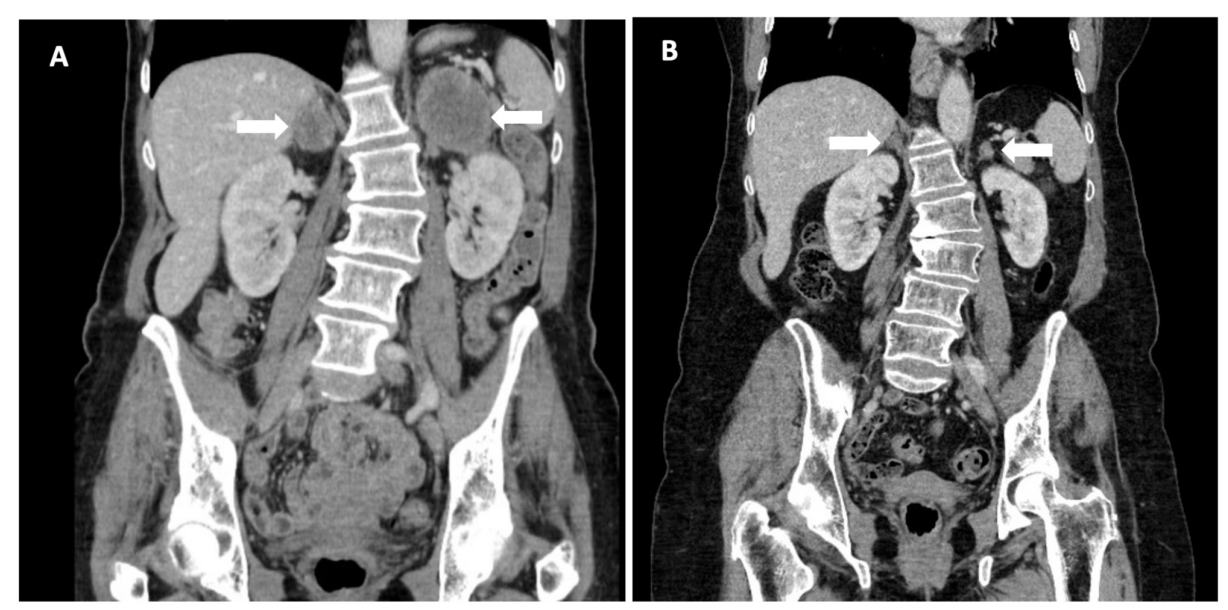

FIGURE 1: Treatment response with nivolumab

A: CT abdomen before initiation of nivolumab demonstrating $6.0 \times 4.4 \mathrm{~cm}$ left adrenal mass and $3.1 \times 2.5 \mathrm{~cm}$ right adrenal mass. B: Near complete resolution of bilateral adrenal masses after 41 months of nivolumab.

\section{Discussion}

Somatic activating $B R A F$ mutations were first described in 2002 and are best recognised in melanoma, identified in up to $66 \%$ of patients [4,5]. BRAF mutations are also found in several other malignancies including $100 \%$ of classic hairy cell leukemia, $50 \%$ of papillary thyroid cancer, and $15 \%$ of colon cancers $[4,6]$. NSCLC genomic studies have found that $1-4 \%$ of all NSCLC, predominantly adenocarcinoma, harbor a $B R A F$ mutation and have similar survival as patients with wild type BRAF [7-10]. ALK-positive and EGFR mutant NSCLC are found more frequently in non-smokers and within the Asian population, while BRAF mutations appear to be more common in current or past smokers and the Caucasian population $[3,7,8,10]$. Over half of NSCLC BRAF mutations are V600E (exon 15), the remainder being non-V600E (exon 11 or 15). G469A accounts for approximately $23 \%$ of NSCLC BRAF mutations, however very little is published on patients whose cancer carries this mutation [10].

Dual $B R A F$ inhibition with dabrafenib and vemurafenib significantly improves overall survival in advanced melanoma [5]. In BRAF V600E mutant metastatic NSCLC, an objective response rate (ORR) of 33-42\% is seen in response to the same treatment, without a similar response observed in non-V600E BRAF carriers [11-13]. Dual $B R A F$ and mitogen-activating protein kinase pathway inhibition, using a combination of dabrafenib and trametinib, respectively, has also been employed in BRAFV600E mutant metastatic NSCLC and found to have a 61-63\% ORR. Unfortunately, BRAF non-V600E mutations were excluded from the trial [14]. With these results the Food and Drug Administration (FDA) approved dabrafenib plus trametinib for BRAF V600E mutant metastatic NSCLC [15]. Although these treatment options are promising, they cannot be applied to patients with BRAF G469A mutations. ICI therapies have now proven to be an effective option for many cancers [16]. However, only a portion of patients respond and experience the benefits of long-term disease control. In patients with NSCLC, the likelihood of response can be partially predicted by the level of PD-L1 expression on cancer cells, but this biomarker has proven far less useful in other malignancies $[17,18]$.

$E G F R$ - or $A L K$-mutation-positive NSCLCs typically have shorter and less complete response to ICI, compared to NSCLC without these oncogenic drivers, even with high PD-L1 expression [2]. This reduction in benefit results in ICI monotherapy being left until late in the treatment algorithm for NSCLC with EGFR- or $A L K$-driven NSCLC and some would extrapolate this concern to other driver mutations. Multiple hypotheses on this diminished response have been proposed, including lower tumor mutation burden or tumorinfiltrating lymphocytes, microenvironment differences, and an alternate escape pathway that is not associated with PD-1/PD-L1 [17]. Most current ICI clinical trials exclude patients with EGFR-mutant- or $A L K$-positive NSCLCs and most new data is achieved through small subgroup analysis and real-world data. Whether BRAF-mutant NSCLCs also have a similar response to ICI remains unclear. Guisier et al. recently considered the efficacy of ICI in Stage IV NSCLC harboring BRAF, HER2, MET mutation, or RET translocation. Twenty-six patients with BRAF V600E mutations demonstrated a $26 \%$ ORR with a median overall survival of 22.5 months. Comparatively, 18 patients had BRAF non-V600 mutations demonstrating a $35 \%$ ORR and 12.0 months median overall survival with ICI, but the specific G469A subgroup was not reported. Patients were predominantly treated with nivolumab; PD-L1 expression was $\geqslant 50 \%$ in $11 \%$ of patients and unknown for 56\% [9]. Our BRAF G469A mutant NSCLC index case experienced a deep and durable response. Given that $B R A F$ mutant NSCLC is found more commonly in current or past smokers, and is associated with high tumor mutation burden, strong response to ICI might be reasonable to expect [1].

\section{Conclusions}


Research is evolving in the evaluation of rare oncogenic drivers. Due to the rarity of specific mutations, accrual of patients in clinical trials is challenging. This profound and prolonged response to an ICI adds to the limited current literature on NSCLC harbouring a BRAF G469A mutation and further suggests that ICI may be of benefit and a reasonable treatment option.

\section{Additional Information \\ Disclosures}

Human subjects: Consent was obtained by all participants in this study. University of Manitoba Health Research Ethics Board issued approval HS23709 (H2020:107). Case Report Title: Immunotherapy benefit in a patient with NSCLC and a rare BRAF mutation The above was approved by Dr. John Arnett, Ph.D., C. Psych., Chair, Health Research Ethics Board, Bannatyne Campus, University of Manitoba on behalf of the committee per your letter received February 20, 2020. Conflicts of interest: In compliance with the ICMJE uniform disclosure form, all authors declare the following: Payment/services info: Dr. Dawe was awarded a salary award by the Manitoba Medical Services Foundation to protect some of his time for research, though this award was not directly to fund the submitted case report. No funding has been received for the case report. Financial relationships: S. Banerji and D.E. Dawe declare(s) personal fees from AstraZeneca. Advisory Board attendeance/Honoraria. S. Banerji and D.E. Dawe declare(s) personal fees from Merck. Advisory Board attendance/Honoraria. S. Banerji and D.E. Dawe declare(s) personal fees from Boehringer-Ingelheim. Advisory Board attendance/Honoraria. S. Banerji declare(s) personal fees from Bayer. Advisory Board attendance/Honoraria. S. Banerji declare(s) personal fees from Bristol-Myers-Squibb. Advisory Board attendance/Honoraria. S. Banerji declare(s) personal fees from GlaxoSmithKline. Advisory Board attendance/Honoraria. S. Banerji declare(s) personal fees from Lilly. Advisory Board attendance/Honoraria. S. Banerji declare(s) personal fees from Novartis. Advisory Board attendance/Honoraria. S. Banerji declare(s) personal fees from Pfizer. Advisory Board attendance/Honoraria. S. Banerji declare(s) personal fees from Roche. Advisory Board attendance/Honoraria. S. Banerji declare(s) personal fees from Takeda. Advisory Board attendance/Honoraria. D.E. Dawe declare(s) a grant from Manitoba Medical Services Foundation. Salary award and research grant. S. Banerji and D.E. Dawe declare(s) a grant from CancerCare Manitoba Foundation. Research grants. D.E. Dawe declare(s) a grant from Canadian Institutes of Health Research. Research grants. Dawe D.E. declare(s) a grant from AstraZeneca. Grant for an unrelated research project. Other relationships: All authors have declared that there are no other relationships or activities that could appear to have influenced the submitted work.

\section{Acknowledgements}

We would like to acknowledge the patient for her willingness to share her experience. Dr. Dawe would like to acknowledge the Manitoba Medical Services Foundation, which awarded him a salary award to protect some of his time for research.

\section{References}

1. Dudnik E, Bshara E, Grubstein A, et al.: Rare targetable drivers (RTDs) in non-small cell lung cancer (NSCLC): outcomes with immune check-point inhibitors (ICPi). Lung Cancer. 2018, 124:117-24. 10.1016/j.lungcan.2018.07.044

2. Gainor JF, Shaw AT, Sequist L V, et al.: EGFR mutations and ALK rearrangements are associated with low response rates to PD-1 pathway blockade in non-small cell lung cancer (NSCLC): a retrospective analysis. Clin Cancer Res. 2016, 22:4585-93. 10.1016/j.physbeh.2017.03.040

3. Baik CS, Myall NJ, Wakelee HA: Targeting BRAF-mutant non-small cell lung cancer: From molecular profiling to rationally designed therapy. Oncologist. 2017, 22:786-96. 10.1634/theoncologist.2016-0458

4. Davies H, Bignell GR, Cox C, et al.: Mutations of the BRAF gene in human cancer. Nature. 2002, 417:949-54. 10.1038/nature00766

5. Schadendorf D, van Akkooi ACJ, Berking C, et al.: Melanoma. Lancet. 2018, 392:971-84. 10.1016/S01406736(18)31559-9

6. Gautschi O, Milia J, Cabarrou B, et al.: Targeted therapy for patients with BRAF-mutant lung cancer results from the European EURAF cohort. J Thorac Oncol. 2015, 10:1451-57. 10.1097/JTO.0000000000000625

7. Cardarella S, Ogino A, Nishino M, et al.: Clinical, pathologic, and biologic features associated with BRAF mutations in non-small cell lung cancer. Clin Cancer Res. 2013, 19:4532-40. 10.1158/1078-0432.CCR-130657

8. Paik PK, Arcila ME, Fara M, et al.: Clinical characteristics of patients with lung adenocarcinomas harboring BRAF mutations. J Clin Oncol. 2011, 29:2046-51. 10.1200//CO.2010.33.1280

9. Guisier F, Dubos-Arvis C, Viñas F, et al.: Efficacy and safety of anti-PD-1 immunotherapy in patients with advanced Non Small Cell Lung Cancer with BRAF, HER2 or MET mutation or RET-translocation. J Thorac Oncol. 2020, 10:1016. 10.1016/j.jtho.2019.12.129

10. Kinno T, Tsuta K, Shiraishi K, et al.: Clinicopathological features of nonsmall cell lung carcinomas with BRAF mutations. Ann Oncol. 2014, 25:138-42. 10.1093/annonc/mdt495

11. Planchard D, Kim TM, Mazieres J, et al.: Dabrafenib in patients with BRAF V600E-positive advanced nonsmall-cell lung cancer: a single-arm, multicentre, open-label, phase 2 trial. Lancet Oncol. 2016, 17:642-50. 10.1016/S1470-2045(16)00077-2

12. Hyman DM, Puzanov I, Subbiah V, et al.: Vemurafenib in multiple nonmelanoma cancers with BRAF V600 mutations. N Engl J Med. 2015, 373:726-36. 10.1056/NEJMoa1502309

13. Mazieres J, Cropet C, Montané L, et al.: Vemurafenib in non-small-cell lung cancer patients with BRAF 


\section{Cureus}

V600 and BRAF nonV600 mutations. Ann Oncol. 2020, 31:289-94. 10.1016/j.annonc.2019.10.022

14. Planchard D, Besse B, Groen HJM, et al.: Dabrafenib plus trametinib in patients with previously treated BRAF V600E-mutant metastatic non-small cell lung cancer: an open-label, multicentre phase 2 trial. Lancet Oncol. 2016, 17:984-93. 10.1016/S1470-2045(16)30146-2

15. Odogwu L, Mathieu L, Blumenthal G, et al.: FDA approval summary: dabrafenib and trametinib for the treatment of metastatic non-small cell lung cancers harboring BRAF V600E mutations. Oncologist. 2018, 23:740-5. 10.1634/theoncologist.2017-0642

16. Yang F, Markovic SN, Molina JR, et al.: Association of sex, age, and eastern cooperative oncology group performance status with survival benefit of cancer immunotherapy in randomized clinical trials: a systematic review and meta-analysis. JAMA Network Open. 2020, 3:e2012534.

10.1001/jamanetworkopen.2020.12534

17. Blons H, Garinet S, Laurent-Puig P, Oudart JB: Molecular markers and prediction of response to immunotherapy in non-small cell lung cancer, an update. J Thorac Dis. 2019, 11:25-36. 10.21037/itd.2018.12.48

18. Meyers DE, Banerji S: Biomarkers of immune checkpoint inhibitor efficacy in cancer . Curr Oncol. 2020, 27:S106-S114. 10.3747/co.27.5549 